\section{A new BCR-ABL1 Drosophila model as a powerful tool to elucidate the pathogenesis and progression of chronic myeloid leukemia}

\author{
Roberto Bernardoni,, 1,2,\$,\# Giorgia Giordani, ${ }^{1,3,4, \S}$ Elisabetta Signorino, ${ }^{3, \S}$ \\ Sara Monticelli, ${ }^{1}$ Francesca Messa, ${ }^{1}$ Monica Pradotto, ${ }^{3}$ Valentina Rosso, ${ }^{3}$ \\ Enrico Bracco, ${ }^{5}$ Angela Giangrande,${ }^{6}$ Giovanni Perini, ${ }^{1,2^{*}}$ Giuseppe Saglio ${ }^{3, *}$ \\ and Daniela Cilloni ${ }^{3, *}$
}

${ }^{1}$ Department of Pharmacy and Biotechnology (FABIT), University of Bologna, Italy; ${ }^{2} \mathrm{Health}$ Sciences and Technology - Interdepartmental Center for Industrial Research (HST-ICIR), University of Bologna, Ozzano Emilia, Italy; ${ }^{3}$ Department of Clinical and Biological Sciences, University of Turin, Italy; ${ }^{4}$ Present address: Department of Biological Sciences, School of Applied Sciences, University of Huddersfield, Queensgate, UK; ${ }^{5}$ Department of Oncology, University of Turin, Italy and 'Institut de Génétique et de Biologie Moléculaire et Cellulaire, CNRS/INSERM/ULP 67404 IIIkirch, France

${ }^{\S}$ These authors share first authorship.

"These authors share last authorship.

\section{ABSTRACT}

1 The oncoprotein BCR-ABL1 triggers chronic myeloid leukemia. It is clear that the disease relies on constitutive BCR-ABL1 kinase activity, but not all the interactors and regulators of the oncoprotein are known. We describe and validate a Drosophila leukemia model based on inducible human BCR-ABL1 expression controlled by tissue-specific promoters. The model was conceived to be a versatile tool for performing genetic screens. BCR-ABL1 expression in the developing eye interferes with ommatidia differentiation and expression in the hematopoietic precursors increases the number of circulating blood cells. We show that BCR-ABL1 interferes with the pathway of endogenous $d A b l$ with which it shares the target protein Ena. Loss of function of ena or Dab, an upstream regulator of $\mathrm{dAbl}$, respectively suppresses or enhances both the BCR-ABL1-dependent phenotypes. Importantly, in patients with leukemia decreased human Dab1 and Dab2 expression correlates with more severe disease and Dab1 expression reduces the proliferation of leukemia cells. Globally, these observations validate our Drosophila model, which promises to be an excellent system for performing unbiased genetic screens aimed at identifying new BCR-ABL1 interactors and regulators in order to better elucidate the mechanism of leukemia onset and progression.

\section{Introduction}

Chronic myeloid leukemia (CML) is a clonal myeloproliferative disorder associated with a reciprocal translocation between chromosomes 9 and 22. This process leads to the fusion of the Abelson ( $A B L 1)$ tyrosine kinase gene with the breakpoint cluster region $(B C R)$ sequences generating a fusion gene encoding the constitutively active protein tyrosine kinase BCR-ABL1. Due to its high frequency in CML patients (95\%), the translocation is considered the cytogenetic hallmark of this disease. ${ }^{1,2}$ Although BCR-ABL1 is one of the most studied oncogenic proteins, some molecular mechanisms leading to cellular transformation are still partially unknown. In particular, positive or negative regulators of BCR-ABL1 have not been completely identified. The fruitfly, Drosophila melanogaster, represents a powerful tool for genome-wide genetic analysis and screens, given the functional conservation and sequence homology between human and Drosophila genes. Genome-wide approaches may allow identification of genetic pathways that contribute to disease onset and/or progression without a priori knowledge of the gene function. ${ }^{3}$ The high degree of conservation between human and
Ferrata Storti Foundation

Haematologica 2019

Volume 104(4):717-728

\section{Correspondence:}

DANIELA CILLONI

daniela.cilloni@unito.it

ROBERTO BERNARDONI

roberto.bernardoni@unibo.it

Received: May 21, 2018.

Accepted: November 8, 2018.

Pre-published: November 8, 2018.

doi:10.3324/haematol.2018.198267

Check the online version for the most updated information on this article, online supplements, and information on authorship \& disclosures: www.haematologica.org/content/104/4/717

(C)2019 Ferrata Storti Foundation

Material published in Haematologica is covered by copyright. All rights are reserved to the Ferrata Storti Foundation. Use of published material is allowed under the following terms and conditions:

https://creativecommons.org/licenses/by-nc/4.0/legalcode. Copies of published material are allowed for personal or internal use. Sharing published material for non-commercial purposes is subject to the following conditions:

https://creativecommons. org/licenses/by-nc/4.0/legalcode, sect. 3. Reproducing and sharing published material for commercial purposes is not allowed without permission in writing from the publisher. 
Drosophila Abl (dAbl) proteins and the existence of Drosophila homologs for many proteins that interact functionally with BCR-ABL1 in mammals strongly support the idea that $\mathrm{dAbl}$ and presumably BCR-ABL1 signal transduction pathways could be highly conserved from fly to human. The $d A b l$ gene is expressed at high levels in differentiating neurons and plays an important role in central nervous system, eye and epithelia development, mainly regulating cytoskeleton remodeling. ${ }^{4-6}$ Interestingly, Forgerty and colleagues demonstrated that the neural expression of a chimeric $\mathrm{BCR}-\mathrm{ABL}$ protein carrying the human $\mathrm{BCR}$ fused to $\mathrm{dAbl}$ is able to rescue the $d A b l$ mutant phenotype, suggesting that the chimeric BCR-ABL protein can effectively compensate for lack of dAbl. ${ }^{7}$ To further identify genes and pathways involved in the onset and progression of CML, we developed and validated a genetic model based on transgenic flies that drive inducible human BCR-ABL1 expression under the control of tissue- and stage-specific promoters, providing both an excellent and powerful model to identify novel functional interactors.

\section{Methods}

\section{Generation of BCR-ABL1 transgenic flies}

The $B C R-A B L 1$ coding sequence was amplified by polymerase chain reactions and cloned into the P-element expression vector pKS69. BCR-ABL1 kinase-dead (BCR-ABL1 $1^{\mathrm{KD}}$ ) was obtained through site-directed mutagenesis (Online Supplementary Data). Plasmids were prepared using Oiafilter ${ }^{\mathrm{TM}}$ Plasmid Maxi Kit (Qiagen, Venlo, the Netherlands) and injected in Drosophila embryos (The BestGene, Inc, Chino Hills, CA, USA).

\section{Drosophila stocks}

Fly stocks were obtained from Bloomington Drosophila Stock Center (Department of Biology, Indiana University, Bloomington, IN, USA). RNA interference (RNAi) lines were obtained from the Vienna Drosophila RNAi Center (Vienna, Austria). domeless Gal4 and STAT ${ }^{D N}$ flies were kindly provided by A. Giangrande (IGBMC, Illkirch, France) (Online Supplementary Data).

\section{Immunoblotting}

Adult heads were dissected and homogenized in a protein extraction buffer. For cell lines, $10^{7}$ cells were lysed in RIPA buffer. The following primary antibodies were used: c-Abl (sc23), Dab1 (sc-271136), p-Tyr (sc-7020), GAPDH (sc-137179) (Santa Cruz Biotechnology, Santa Cruz, CA, USA), $\alpha$-tubulin (CP06; Oncogene Research Products, Merck KGaA, Darmstadt, Germany) mouse monoclonal antibodies, BCR (sc-20707) rabbit polyclonal antibody (Santa Cruz Biotechnology) and mouse 5G2 anti-Enabled supernatant (Developmental Studies Hybridoma Bank - DSHB, University of Iowa, IA, USA). For immunoprecipitation, $1 \mathrm{mg}$ of total protein extract was incubated with anti-Enabled supernatant and subsequently with protein A sepharose (Amersham Bioscience, GE Healthcare, Waukesha, WI, USA) (Online Supplementary Data).

\section{Fluorescent Immunolabeling Fly eye primordium}

Eye imaginal discs were dissected from third instar larvae, fixed in $4 \%$ paraformaldehyde, permeabilized with $0.3 \%$ Triton X-100, labeled with the rat anti-Elav 7E8A10 supernatant
(DSHB), incubated with a Cy3-conjugated anti-rat secondary antibody (Jackson Immunoresearch, Newmarket, UK) and exposed to HOECHST (Sigma-Aldrich Corp., St. Louis, MO, USA) before mounting in Fluormount-G (Electron Microscopy Sciences, Hatfield, PA, USA) (Online Supplementary Data).

\section{Primary cells}

The protocol was approved by the local ethics committee (approval n. 212/2015). White blood cells $\left(10^{5}\right)$ were obtained from peripheral blood. Immunofluorescence was performed as previously described ${ }^{8}$. Mouse anti-Dab1 and anti-Dab2 primary antibodies (sc-271136 and sc-136963, Santa Cruz Blotechnology) and anti-mouse Alexa Fluor 568 secondary antibody (Molecular Probes-Invitrogen, ThermoFisher Scientific, Waltham, MA, USA) were used (Online Supplementary Data).

\section{Genetic analysis}

Eye

Flies carrying gmrGal4 or sevGal4 driver constructs were crossed to the UAS-BCR-ABL1 transgenic lines. To analyze the phenotype, flies from a recombinant line carrying both gmrGal4 and UAS$B C R-A B L 1$ on the third chromosome (gmrGal4,UAS-BCR-ABL1 $4 M / T M 3)$ were crossed to lines carrying single gene mutations, deficiencies or RNAi constructs. Fifteen to 30 F1 flies from three independent crosses were classified into three phenotypic classes described in the Results section.

\section{Melanotic nodules}

domelessGal4-driven BCR-ABL1 expression was controlled with the TARGET system ${ }^{9,10}$ (Online Supplementary Data). We performed conditional expression in the medullary zone of the lymph gland starting at different stages during larvae development by moving the animals from $18^{\circ} \mathrm{C}$ to $29^{\circ} \mathrm{C}$. Analysis of the melanotic nodule phenotype and temperature shift experiments were performed as previously described. ${ }^{11}$

\section{RNA extraction and quantitative analysis}

RNA was extracted using standard procedures. Expression levels of Dab1 and Dab2 were evaluated by real-time polymerase chain reaction using specific on-demand kits (Hs00245445_m1 for ABL1, Hs00221518_m1 for Dab1, Hs00184598_m1 for Dab2, Applied Biosystems, ThermoFisher Scientific) according to published methods. ${ }^{12}$

\section{Results}

\section{Expression of human BCR-ABL1 affects eye cell differentiation}

The aim of this work was to set up a CML Drosophila model based on the expression of a completely human BCR-ABL1 fusion protein. Available Drosophila genetic tools allow expression of proteins of interest in developing eye cells, often inducing viable and visible phenotypic traits that can be used as a bait in genetic screening. The Drosophila eye differentiates during the third instar larva (L3) from the eye imaginal disc, a monolayer epithelium that is accessible to dissection. We generated several stable transgenic fly lines to express BCR-ABL1 protein using the yeast Gal4/UAS (Upstream Activating Sequence) transcriptional regulation system controlled by a gene promoter active in specific tissues and stages (Gal4 drivers). ${ }^{13}$ BCR-ABL1 expression was first triggered with the sevenlessGal4 (sevGal4) construct that drives 
high levels of expression in some but not all photoreceptors ${ }^{14}$ producing a mild rough eye similar to the one observed by Fogerty ${ }^{7}$ (Figure 1A-E). This suggests that BCR-ABL1 interferes with eye development as described for the human/fly chimera. To drive BCR-ABL1 expression in more eye cells, we used the glass multimer reporterGal4 (gmrGal4) driver, active in all cells committed to differentiation and located posteriorly to the morphogenetic furrow, ${ }^{15}$ the cell indentation crossing the eye pri- mordium from posterior to anterior (Figure $1 \mathrm{~N}, \mathrm{O}$ ). BCRABL1 expression in these cells produced a severe "glazed" eye phenotype (Figure 1F-J, Online Supplementary Figure $S 1 A, B, H, I)$. The regular structure of the eye was almost completely lost: ommatidia, the functional units of the eye, failed to differentiate and were no longer distinguishable. The eye was smaller, bar-shaped and misplaced extra sensory bristles appeared in the dorsal region (Figure 1H-J). Western blot

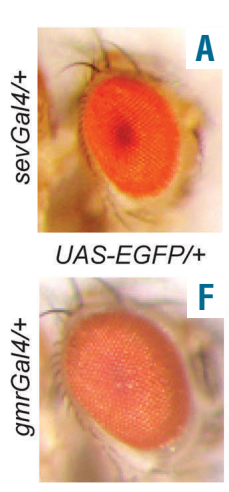

UAS-EGFP/+

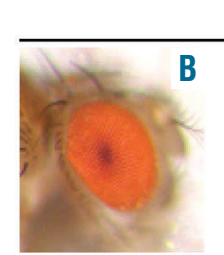

$1 M$

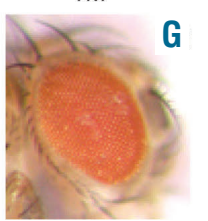

$1 M$

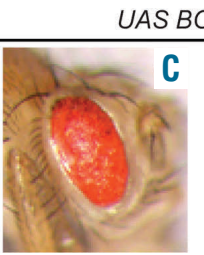

$3 M$

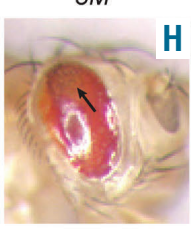

$3 M$

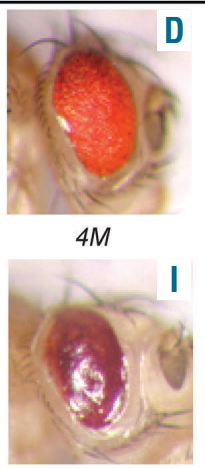

$4 M$

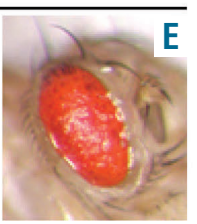

$7 M$

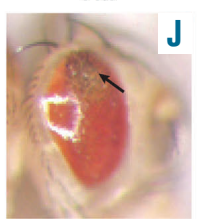

$7 M$

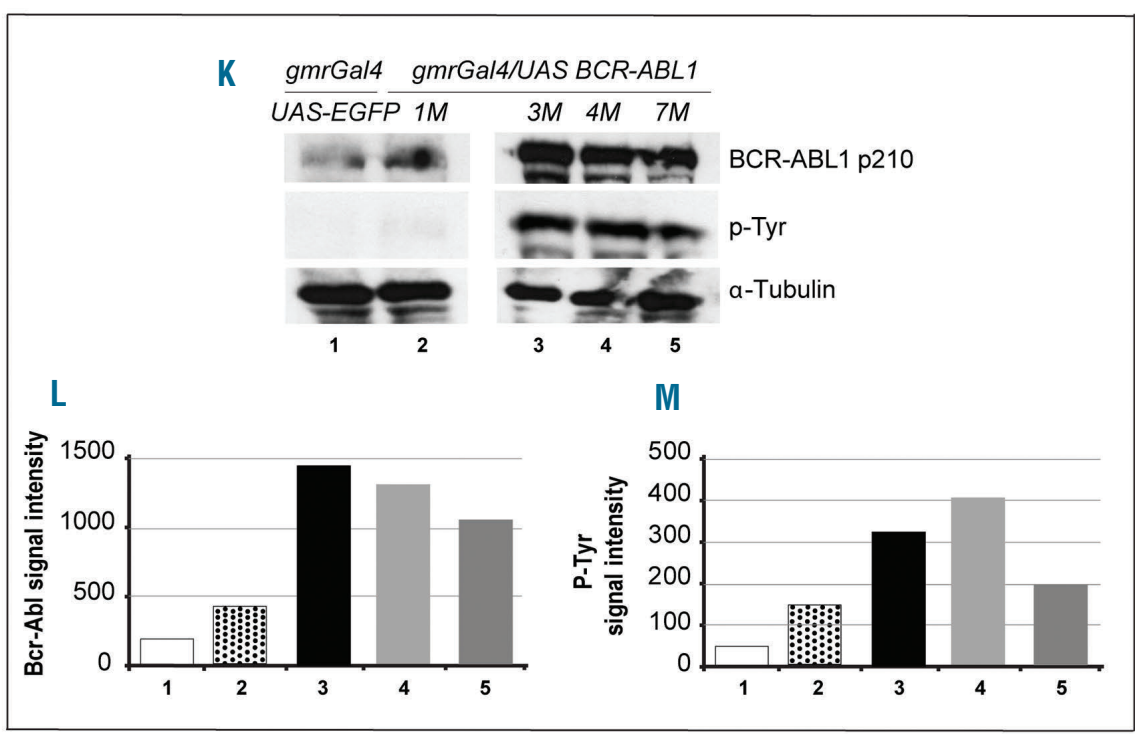

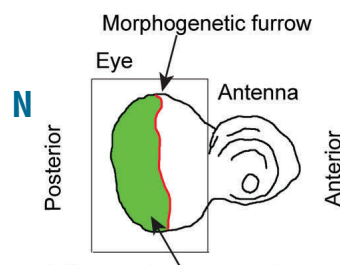

Differentiating retina cells

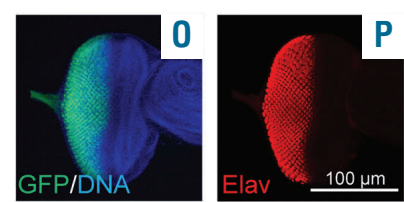

UAS-EGFP/+; gmrGa/4/+

UAS BCR-ABL1

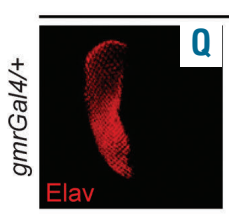

$1 M$

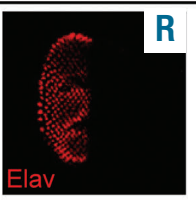

$3 M$

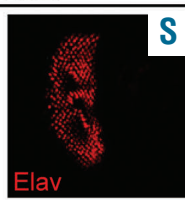

$4 M$

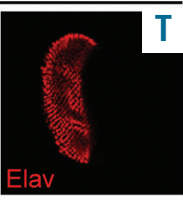

$7 M$
Figure 1. BCR-ABL1 expression in the developing eye cells affects photoreceptor differentiation. (A-E) Adult eyes expressing EGFP (A) or BCR-ABL1 in four independent transgenic lines, 1M (B), 3M (C), 4M (D), or 7M $(\mathrm{E})$, in a subset of differentiating photoreceptor cells under the control of the sevenlessGal4 driver construct. (C-E) High levels of BCR-ABL1 induce a "rough" eye phenotype due to impairment of cell differentiation. (F-J) Adult eyes expressing EGFP (F) or BCR-ABL1 (G-J) in all differentiating eye cells under the control of the gmrGal4 driver construct. (H-J) BCR-ABL1 expressed at high level in all differentiating eye cells profoundly disrupts ommatidia development inducing a "glazed" phenotype, depigmented area and the appearance of extra bristles (black arrows). (K-M) Quantification of BCRABL1 expression (K,L) and tyrosine-phosphorylation $(\mathrm{K}, \mathrm{M})$ in protein extracts from adult heads of flies expressing either EGFP (lane 1) or BCR-ABL1 in independent transgenic fly lines (lanes 2-5). The protein extracts were probed with antibodies raised against BCR, phosphorylated tyrosine residues ( $p$-Tyr) or $\alpha$-tubulin as the loading control. (N) Schematic of the eye-antenna imaginal disc from a late third instar larva; the positions of the eye and antenna primordia and of the morphogenetic furrows are indicated. The eye imaginal disc area posterior to the morphogenetic furrow, made of cells committed to terminal differentiation, is indicated in green. The thin black square indicates the region of interest shown in panels O-T. $(O, P)$ Eye imaginal disc from wildtype late third instar larvae expressing EGFP under the control of the gmrGal4 driver in cells posterior to the morphogenetic furrow and expressing the pan-neuronal marker Elav in cells committed to terminal differentiation. (Q-T) Elav expression in eye imaginal discs from third instar larvae of the four independent transgenic lines that express BCR-ABL1 under the control of the gmrGal4 driver construct. BCR-ABL1 expression reduces the number of differentiated photoreceptors as indicated by the decrease of Elav-expressing cells. 
analysis demonstrated that the severity of the phenotype correlated with the amount and phosphorylation of BCR-ABL1 protein (Figure 1K-M): indeed the low level of BCR-ABL1 expression observed in line $1 \mathrm{M}$ (Figure 1K-M) resulted in a very mild phenotype (Figure 1G). To better understand the origin of the phenotype, we analyzed the expression of the pan-neuronal and eye photoreceptor marker Elav ${ }^{16}$ in eye imaginal discs expressing BCRABL1. The typical Elav ${ }^{+}$photoreceptor clusters (Figure 1P) were reduced in number and altered in BCR-ABL1expressing flies and this correlated with the described defects of the eye's ordered structure (Figure 1P-T). To assess whether the phenotype depends on BCR-ABL1 kinase activity, we generated transgenic flies to express a kinase-dead mutant BCR-ABL1. gmrGal4-driven expression of the mutant protein did not affect eye development, indicating that the BCR-ABL1 phenotype requires the enzymatic activity of the oncoprotein (Online Supplementary Figure S1A-C,H).

\section{Expression of human BCR-ABL1 interferes with eye development by altering $d A b$ signaling}

To better understand the consequences of BCR-ABL1 overexpression in the eye, we investigated whether the human oncoprotein could activate the endogenous pathway regulated by the Drosophila Abl kinase (dAbl). To quantify the phenotype we classified BCR-ABL1 eyes (line $4 \mathrm{M}$ ) into three phenotypic classes. Class 0 represents the most frequent "glazed" phenotype. Class +1 is less severe: the eye is bigger and more prominent, and some ommatidia can be observed. Class -1 is more severe, being characterized by a less differentiated eye with evident lack of pigmentation in the most posterior region (Figure 2A). Interestingly, phenotype expressivity did not change comparing gmrGal4,UAS-BCR-ABL1 $4 M$ animals with gmrGal4,UAS-BCR-ABL1 4M; UAS-EGFP (Figure 2B, Online Supplementary Figure $S 1 H, I$ ) indicating that a single gmr Gal4 copy does not express a Gal4 limiting amount that could be titrated by increasing the number of UAS sequences. Since overexpression of $d A b l$ (UAS-Abl) induces a very mild rough eye phenotype (Online Supplementary Figure $S 1 A, B, G$ ), we investigated whether it could enhance the BCR-ABL1 phenotype. We observed a worsening of the phenotype: all of the eyes belonged to class -1 , showing smaller eyes and more evident loss of pigmentation (Figure 2B, Online Supplementary Figure $S 1 G, H, N)$. We then investigated whether $d A b l$ loss of function (LOF) could suppress the BCR-ABL1 phenotype. gmrGal4,UAS-BCR-ABL1 $4 M$ animals heterozygous for a $d A b l$ hypomorphic recessive lethal allele $\left(\mathrm{Abl}^{1 /+}+\right)$ showed a very mild phenotypic suppression but were not statistically different from controls (Figure 2B, Online Supplementary Figure $S 1 E, H, L)$. However, dAbl downregulation through RNAi ( $A b l$ $R N A i$ ) or expression of a dominant negative kinasedefective dAbl (UAS-Abl $\left.{ }^{\mathbb{4} 17 \mathrm{M}}\right)$ induced a significant suppression of the BCR-ABL1 phenotype (Figure 2B, Online Supplementary Figure $S 1 D, F, H, I, K, M)$. Interestingly, we observed that animals expressing either $A b l-R N A i$ or $U A S-A b l$ or $U A S-A b k^{k 417 N}$ showed a similar mild disorganization of the ommatidia (Online Supplementary Figure $S 1 A, B, D, F, G)$ suggesting that the pathway activated by $\mathrm{dAbl}$ is indeed implicated in eye development. Furthermore, the genetic interactions between BCRABL1 expression and $d A b l$ loss or gain of function sug- gest that $\mathrm{dAbl}, \mathrm{dAb} \mathrm{K}^{\mathrm{K} 11 \mathrm{~N} N}$ and overexpressed BCR-ABL1 could compete for common binding targets. To confirm that BCR-ABL1 overexpression affects eye development by altering dAbl signaling cascade, we analyzed whether BCR-ABL1 could functionally interact with components of the dAbl pathway. In detail, we focused on four genes whose LOF mutations genetically interact with a $d A b l$ mutant phenotype. Mutations of prospero (pros), a transcription factor that regulates neuronal differentiation ${ }^{17}$, failed axon connections ( $f a x$ ), implicated both in neurogenesis and axonogenesis ${ }^{18}$ and Disabled (Dab) that regulates cellular localization of $d A b l^{19}$, enhance the mutant $d A b l$ phenotype. Moreover, enabled (ena) gene mutations suppress a $d A b l$ mutant phenotype. ${ }^{20}$ Interestingly, we found that either a deletion or a mutant allele of pros (Figure 2C, Online Supplementary Figure S2A-C) and fax (Figure 2D, Online Supplementary Figure $S 2 A, D, E)$ was able to enhance the BCR-ABL1 phenotype. Moreover, although the insertional Dab ${ }^{\mathrm{EY} 10190}$ allele did not change the BCRABL1 phenotype significantly, a deletion uncovering the Dab locus enhanced it (Figure 2E, Online Supplementary Figure $S 2 A, F, G)$, confirming that BCR-ABL1 expression alters eye development likely by interacting with components of the $\mathrm{dAbl}$ pathway.

\section{BCR-ABL1 expression increases phosphorylation of the dAbl substrate Ena}

A genetic screen had previously identified an ena LOF allele as a suppressor of the recessive lethality due to dAbl LOF mutations. ${ }^{20}$ Ena is a cytoskeletal regulator that facilitates actin polymerization. ${ }^{21}$ Its cellular localization depends on $\mathrm{dAb}^{5,20,22}$ and it is phosphorylated by both human and Drosophila Abl. ${ }^{23,24}$ Heterozygosis of a LOF ena allele or of an ena deletion suppressed the BCR-ABL1 phenotype (Figure 2F, Online Supplementary Figure $S 2 A, J, K)$. ena silencing with two independent constructs (ena-RNAi), induced a size increase and strong decrease of depigmented tissue in eyes expressing BCR-ABL1 (Figure 3A, Online Supplementary Figure S2A,L,M). Consistently, the analysis of Elav expression highlighted a more correct organization of photoreceptor clusters (Figure 3B-E). Furthermore, we looked at tyrosine-phosphorylation of the endogenous Ena. Flies expressing BCR-ABL1 showed increased levels of Ena tyrosinephosphorylation (Figure 3F,H) even after Ena immunoprecipitation (Figure $3 \mathrm{G}, \mathrm{H}$ ) suggesting that Ena might be phosphorylated by BCR-ABL1. Taken together our data indicate that alteration of several components of the dAbl pathway could be important for the mechanism by which BCR-ABL1 overexpression affects eye development, likely phosphorylating conserved targets in fly eye cells.

\section{A component of the BCR-ABL1-activated pathway in human leukemia modulates the eye phenotype in Drosophila}

To further assess the effectiveness of the model, we investigated whether a Drosophila homolog of a gene known to be involved in BCR-ABL1 signaling in human leukemia was also able to modulate the BCR-ABL1 phenotype. Signal transducer and activator of transcription 5 (STAT5) is a transcription factor activated in response to cytokines and its role in malignant transformation is well established. ${ }^{25}$ Several studies showed that BCR-ABL1 induces phosphorylation and constitutive activation of 
A

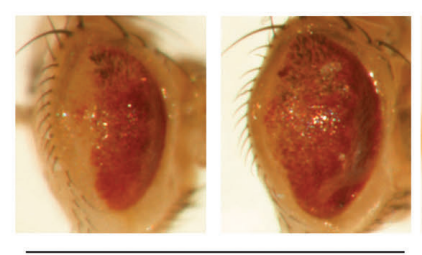

$-1$

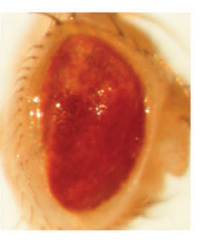

0

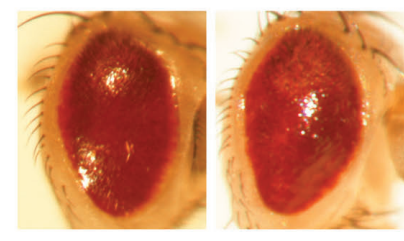

$+1$

Abelson
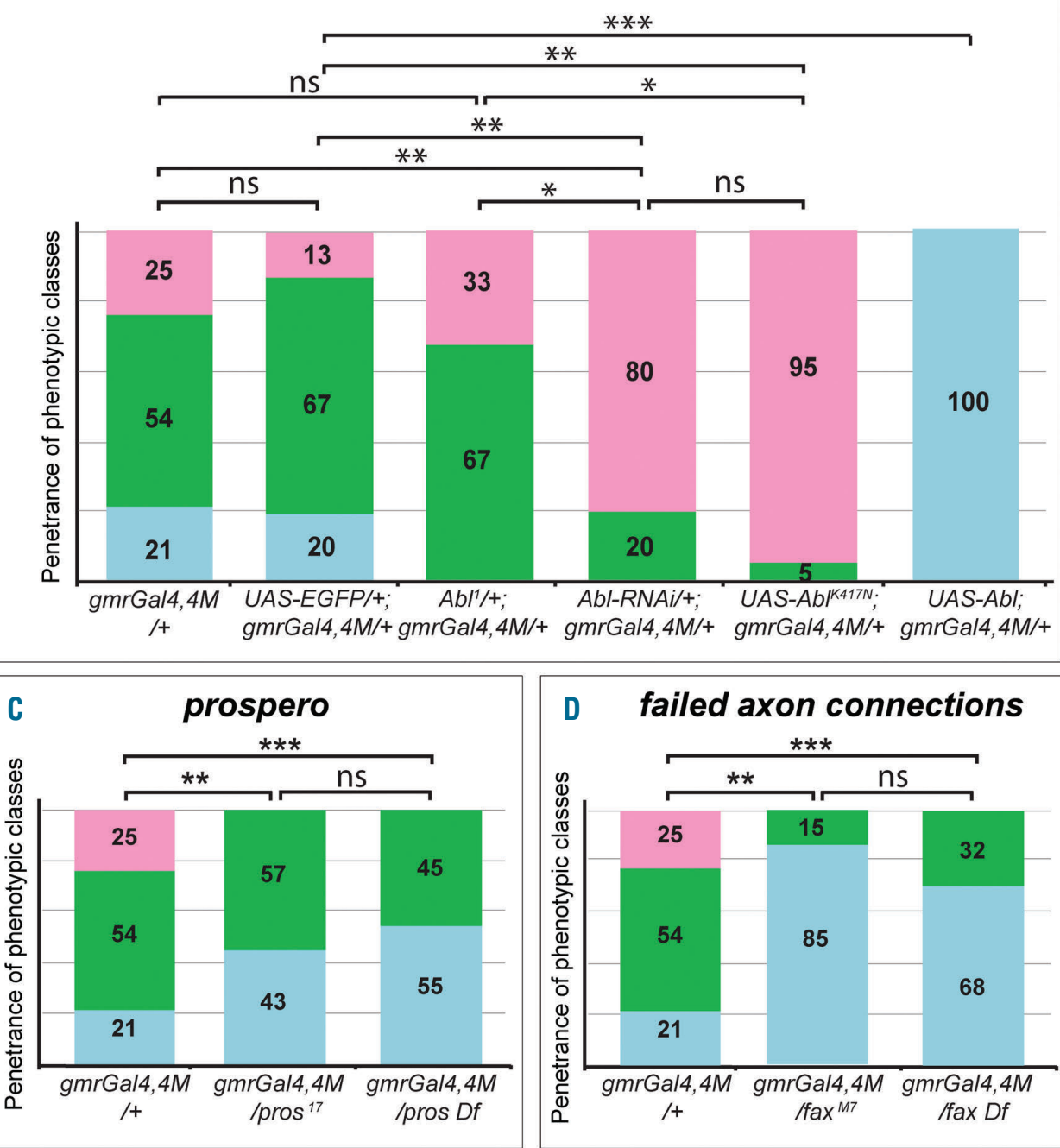

D failed axon connections

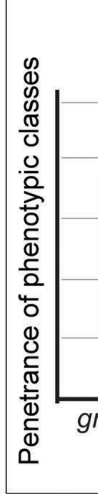

F

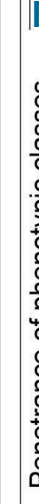

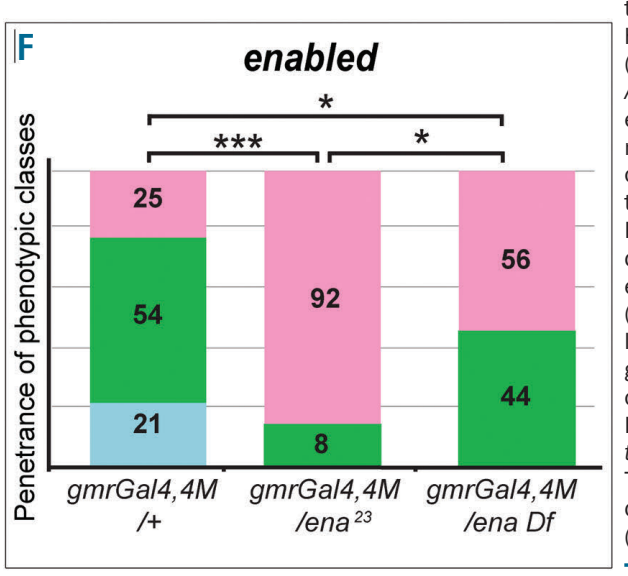

Figure 2. BCR-ABL1 expression affects ommatidia development by altering the dAbl signaling pathway. (A) Adult eyes showing the phenotypic classes used to quantify the severity of the variable phenotype due to BCR-ABL1 expression in eye cells committed to terminal differentiation. Posterior is on the left. Class 0 (green) corresponds to the average phenotype shown by gmrGal4, UAS-BCR-ABL1 $4 M$ flies; the ommatidia are almost totally absent, the eye depigmented region is very small and the eye appears flatter than that of wild-type eyes. Class -1 (pale blue) corresponds to more severe phenotypes: the eyes are even flatter than class 0 eyes and the depigmented area is enlarged including a dorso-ventral sector in the most posterior region of the eye. Class +1 (pink) corresponds to less severe phenotypes: a few ommatidia are visible, the eyes are more bulging and the depigmented area is absent indicating better eye cell differentiation. (B-F) Adult eyes from flies of the indicated genotypes were classified to evaluate the frequency of the three phenotypic classes. (B) A piled histogram chart showing the frequencies of the different phenotypic classes in flies expressing BCR-ABL1 (gmrGal4,4M/+), co-expressing BCR-ABL1 and the EGFP (UASEGFP/+;gmrGal4,4M/+), expressing BCR-ABL1 but having a partial loss of the endogenous $A b l$ gene through the heterozygous $A b l^{1} \quad$ mutation $\left(A \mathrm{Cl}^{1} /+;\right.$ gmrGal4,4M/+), RNAi targeting Abl (Abl-RNAi/+;gmrGal4,4M/+), expression of a dominant negative $\mathrm{dAbl}$ mutant $\left(U A S-A b l^{K 417 N} ; g m r G a l 4,4 M /+\right)$ or overexpression of the wild-type Abl protein (UAS-Abl;gmrGal4,4M/+). (C-F) Piled histogram charts showing the frequencies of the three phenotypic classes in flies expressing BCR-ABL1 (gmrGal4,4M) and heterozygous for a loss of function allele or for a deletion of genes that behave as genetic modifiers of the embryonic lethality due to $A b l$ LOF: prospero (C), failed axon connection $(\mathrm{D})$, Disabled $(\mathrm{E})$ and enabled $(\mathrm{F})$. The statistical comparisons were conducted using a Mann-Whitney test $(* P<0.05, * * P<0.01, * * * P<0.001)$. 

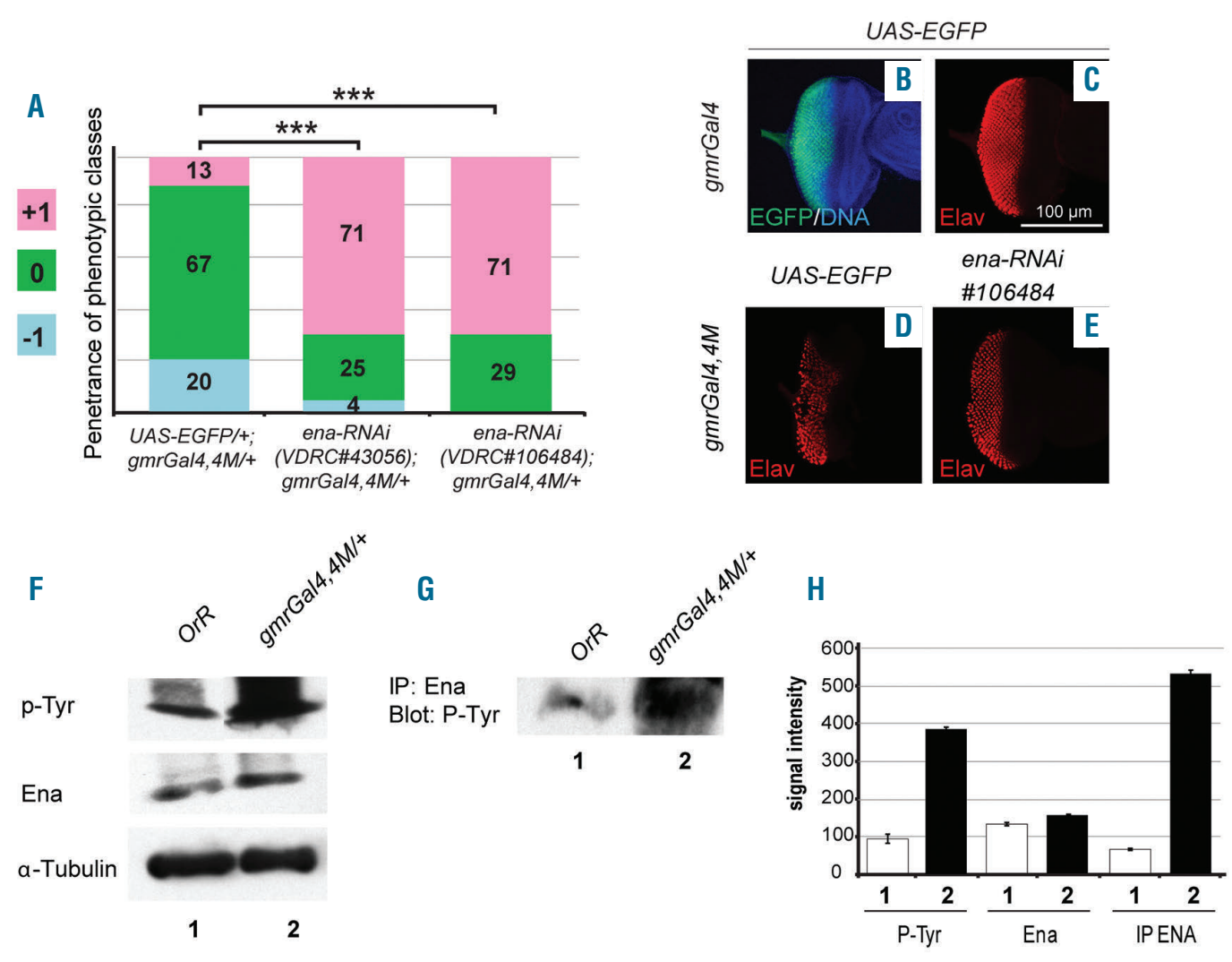

Figure 3. Ena loss of function suppresses the eye phenotype due to BCR-ABL1 expression which increases phosphorylation of the dAbl target Ena. (A) Piled histogram chart showing the frequencies of the three phenotypic classes in flies co-expressing BCR-ABL1 (gmrGal4,4M) and EGFP (UAS-EGFP/+;gmrGal4,4M/+), or one of two independent ena-RNAi lines (VDRC\#43056 and VDRC\#106484). (B,C) Eye imaginal discs from wild-type late third instar larvae expressing EGFP under the control of the gmrGal4 driver in cells posterior to the morphogenetic furrow (B) and expressing the pan-neuronal marker Elav in cells committed to terminal differentiation (C). (D,E) Elav expression in eye imaginal discs from late third instar larvae expressing BCR-ABL1 (D) or larvae co-expressing BCR-ABL1 and ena-RNAi (E) under the control of the gmrGal4 driver construct. BCR-ABL1 expression reduces the number of differentiated photoreceptors, as indicated by a decrease of Elav-expressing cells, and ena downregulation suppresses this phenotypic trait. $(\mathrm{F}-\mathrm{H})$ Quantification of Ena expression and tyrosine-phosphorylation in protein extracts ( $\mathrm{F}$ ) or Enaimmunoprecipitated proteins (G) from the heads of adult flies expressing either EGFP (lane 1) or BCR-ABL1 (lane 2). Independent loads of equal amount of protein extracts or Ena-immunoprecipitated proteins were probed with antibodies raised against phosphorylated tyrosine residues ( $p$-Tyr), Ena or $\alpha$-tubulin as loading control. (H) Average signal intensity from replica of the experiment shown in $(F)$ and $(G)$. Ena immunoprecipitation and probing for tyrosine-phosphorylation confirmed the increase of Ena tyrosine-phosphorylation in animals expressing BCR-ABL1. The statistical comparisons in $(A)$ were conducted using the Mann-Whitney test $(* P<0.05$, $* * P<0.01, * * * P<0.001)$.

STAT5, hindering apoptosis in leukemic cells. ${ }^{26}$ The JAK/STAT pathway is required during Drosophila eye morphogenesis and larval hematopoiesis. ${ }^{27,28}$ Interestingly, loss of STAT92E function $\left(S T A T 92 E^{06346}\right.$ ), the fly counterpart of STAT5, induced strong suppression of the BCR-ABL1 phenotype (Figure 4, Online Supplementary Figure $S 3 A, B)$. Flies coexpressing a STAT dominant negative allele $\left(S T A T^{\mathrm{DN}}\right)$ and BCR-ABL1 showed an even weaker phenotype (Figure 4A, Online Supplementary Figure $S 3 A, C$ ) confirming that STAT is involved in the BCR-ABL1-activated pathway in the Drosophila eye.

The human homologs of Disabled, Dab1 and Dab2, are altered in patients with chronic myeloid leukemia

To better explore the efficacy of the model we analyzed the Disabled gene that encodes for an adaptor protein acting downstream of many receptor tyrosine kinases. ${ }^{17,29}$ In the embryo Dab LOF disrupts the intracellular localization of $\mathrm{dAbl}$ and consequently that of phosphorylated Ena and F-Actin accumulation. ${ }^{30}$ In the fly eye we observed an enhancement of the BCR-ABL1 phenotype in animals heterozygous for a Dab deletion. Thus, we further reduced Dab function by gene silencing.

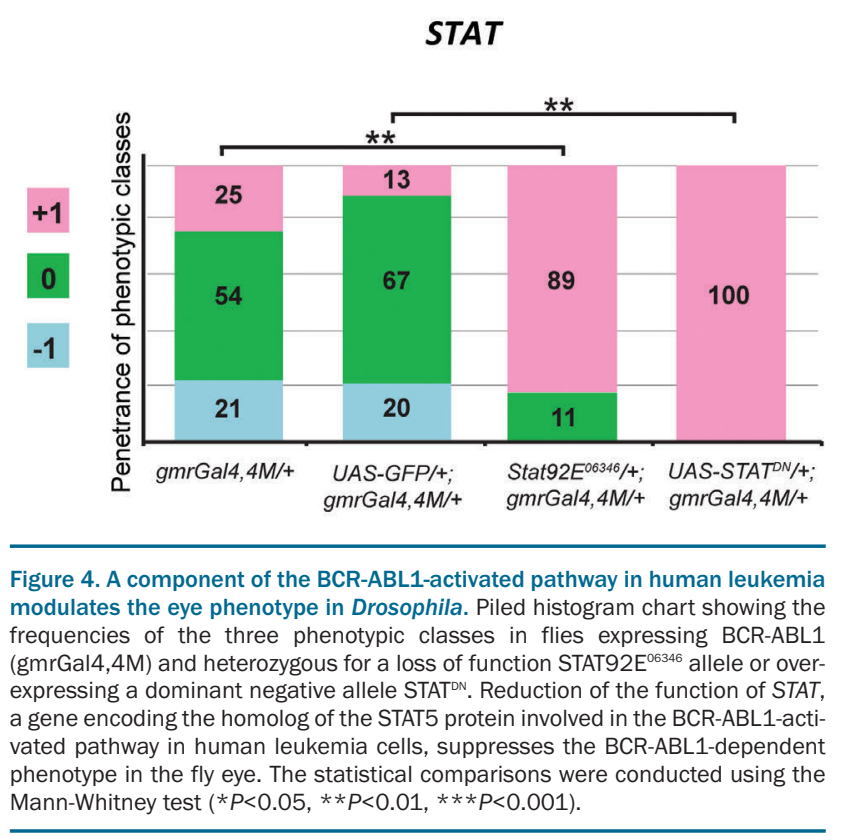


Interestingly, two independent RNAi lines worsened the BCR-ABL1 phenotype more than the Dab deletion did (Figures 2E and 5A, Online Supplementary Figure S2A, F-I): most of the eyes were smaller and showed depigmented scar-like tissue (Online Supplementary Figure S2A, F, $H, I$ ). Consistently, alterations of the ommatidia clusters, revealed by Elav expression, worsened compared to those of the control (Figure 5B-E). To establish whether Dab might have a role in CML we analyzed the two human counterparts of Disabled, Dab1 and Dab2 in human primary cells. Dab1 is a large, common fragile site gene and the Dab1 protein acts as a signal transducer that interacts with many receptor tyrosine kinase pathways. ${ }^{31}$ Dab2 encodes for an adaptor protein implicated in growth factor signaling, endocytosis, cell adhesion, hematopoietic cell differentiation and cell signaling of various receptor tyrosine kinases. ${ }^{32}$ The expression of both genes is often decreased in many human solid cancers, suggesting their possible role in oncogenesis., ${ }^{31,33}$ Interestingly, quantitative real-time polymerase chain reaction analysis revealed a significant downregulation of both genes in CML patients at diagnosis compared to controls in peripheral blood or bone marrow samples (Figure 6A,G). Analysis of bone marrow samples from CML patients during molecular remission showed increased levels of expression of both Dab1 (Figure 6B) and Dab2 (Figure $6 \mathrm{H}$ ) with respect to the levels in treatment-resistant patients. Moreover, immunofluorescence assays demonstrated a significant down-modulation of both proteins in peripheral blood samples at diagnosis compared to the levels in controls or patients in molecular remission (Figure 6C,D,I,J). Finally, transfection experiments in K562 cells using a plasmid carrying the whole Dab1 coding sequence demonstrated that reactivation of Dab1 expression reduced cell proliferation (Figure 6E,F).

\section{BCR-ABL1 expression impairs Drosophila blood cell homeostasis}

To further confirm the efficacy of the model, we inves- tigated the effects of BCR-ABL1 expression in the lymph gland, the hematopoietic organ of the larva. The lymph gland begins to develop in the embryo ${ }^{34}$ and grows up from multipotent progenitor cells (prohemocytes) that proliferate and enter a quiescent phase during the second instar (L2). During the third instar (L3) some prohemocytes start to proliferate again and differentiate. The lymph gland breaks apart at the beginning of metamorphosis releasing differentiated blood cells (hemocytes) into the hemolymph, the Drosophila blood. ${ }^{35,36}$ During the L3, three functional regions can be distinguished in the lymph gland: $:^{37}$ the medullary zone, populated by prohemocytes; the posterior signaling center that regulates the exit of prohemocytes from quiescence; and the cortical zone, made up of differentiating hemocytes. . $^{38,39}$ The lymph gland can break up prematurely in late-L3 if the number of differentiating hemocyte increases. As a reaction to excessive hematopoiesis, the hemocytes aggregate and a spontaneous process of melanization takes place inducing the formation of melanotic nodules. ${ }^{11,36,40,41}$ Constitutive BCR-ABL1 expression under the control of the domelessGal4 (domeGal4) driver, active in the medullary zone of the lymph gland, ${ }^{11,42}$ is lethal (data not shown). To overcome this problem, we repressed expression of BCR-ABL1 by co-expressing a heat-sensitive mutant of the Gal4 repressor Gal80 (tubGal80 ${ }^{T S}$ ) until larvae reached the desired instar (TARGET system). ${ }^{10}$ While BCR-ABL1 expression from the first instar (L1) induced lethality (data not shown), expression from the L2 allowed larvae to survive and to develop melanotic nodules at L3 (Figure 7A,B). This suggests that BCR-ABL1 expression in the medullary zone precursors might induce an increase of circulating hemocytes (Figure 7C). When compared to controls (Figure 7A), $45 \%$ of domeGal4,BCR-ABL1 3M,tubGalso ${ }^{T S}$ larvae showed two to three small melanotic nodules (Figure $7 \mathrm{~B}, \mathrm{C})$. This correlates with an increased number of circulating hemocytes in hemolymph preparations (Figure 7D). BCRABL1 expression starting from the early L3 did not show
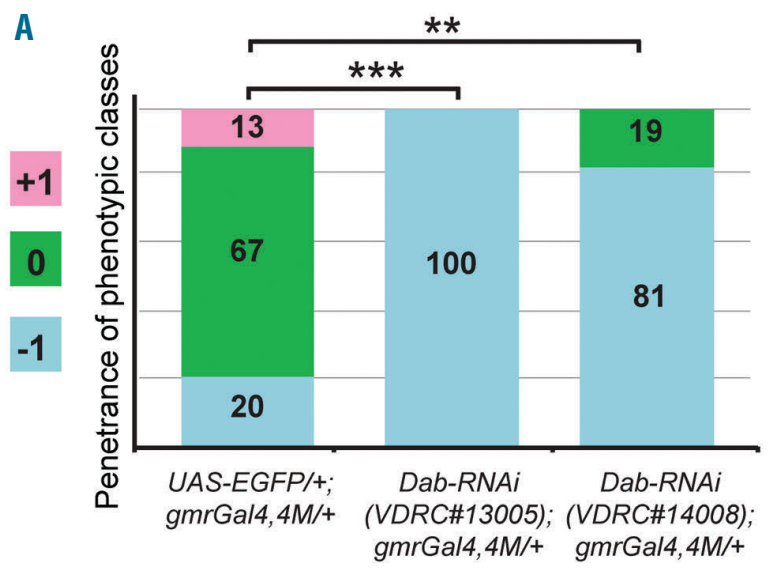

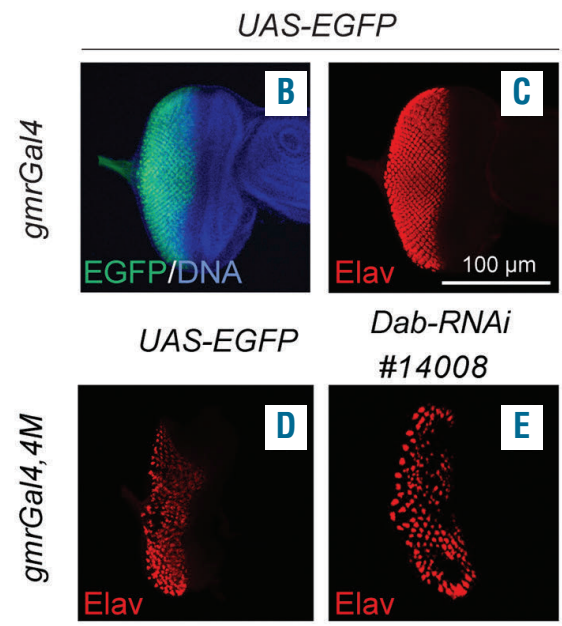

Figure 5. Dab downregulation enhances the eye phenotype due to BCR-ABL1. (A) Piled histogram chart showing the frequencies of the three phenotypic classes in flies co-expressing BCR-ABL1 (gmrGal4,4M) and EGFP (UAS-EGFP/+;gmrGal4,4M/+), or one of two independent Dab-RNAi constructs (VDRC\#13005, VDRC\#14008). $(B, C)$ Eye imaginal discs from wild-type late third instar larvae expressing EGFP under the control of the gmrGal4 driver in cells posterior to the morphogenetic furrow and expressing the pan-neuronal marker Elav in cells committed to terminal differentiation. (D,E) Elav expression in eye imaginal discs from late third instar larvae expressing BCR-ABL1 (D) or larvae co-expressing BCR-ABL1 and Dab-RNAi (E) under the control of the gmrGal4 driver construct. BCR-ABL1 expression reduces the number of differentiated photoreceptors, as indicated by a decrease of Elav-expressing cells, and Dab downregulation enhances this phenotypic trait. The statistical comparisons were conducted using a Mann-Whitney test $(* P<0.05, * * P<0.01, * * * P<0.001)$. 
any significant phenotype (Figure 7C), indicating that only when BCR-ABL1 is expressed when prohemocytes enter quiescence is it able to increase hematopoiesis. Consistently, constitutive expression of the kinase-dead mutant $B C R-A B L 1^{K D}$ did not induce any significant phenotype (Figure 7C). Since $d A b l$, like $D a b$ and ena, is expressed in the lymph gland, ${ }^{43}$ we assessed whether decreased $d A b l$ function is able to rescue the phenotype. We co-expressed BCR-ABL1 and Abl-RNAi, and observed a significant decrease of the phenotype penetrance (Figure 7E). We then investigated whether Dab or ena downregulation interacts genetically with BCR-ABL1 expression during hematopoiesis as well. Dab-RNAi in the medullary zone starting from L2 was able to enhance the melanotic nodule phenotype, inducing a significant increase of the penetrance (Figure 7F). Consistently, larvae co-expressing Dab $(U A S-D a b)$ and BCR-ABL1 in the medullary zone starting from L2 showed phenotypic suppression (Figure 7F).

A

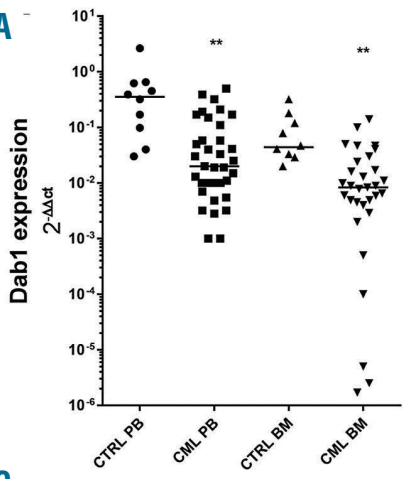

C

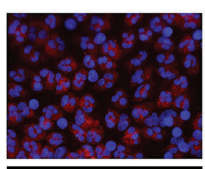

CTRL

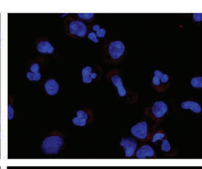

CML diagnosis

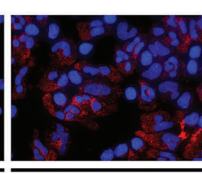

CML MR
B
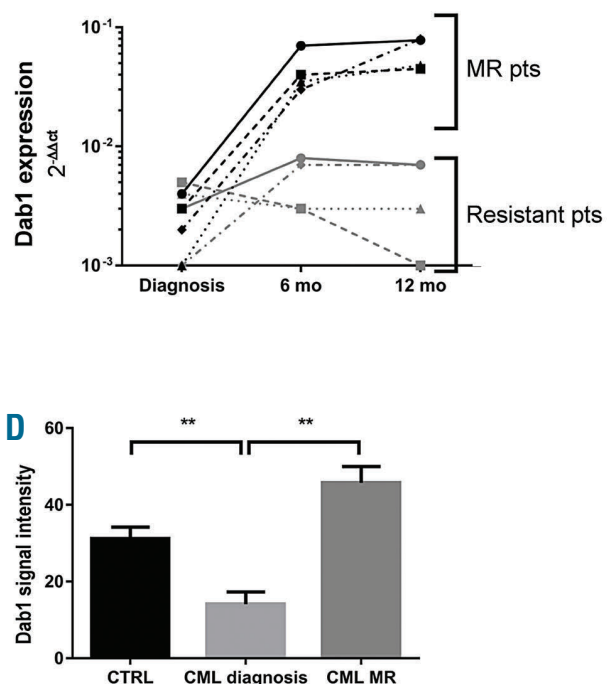

$\mathbf{F}$

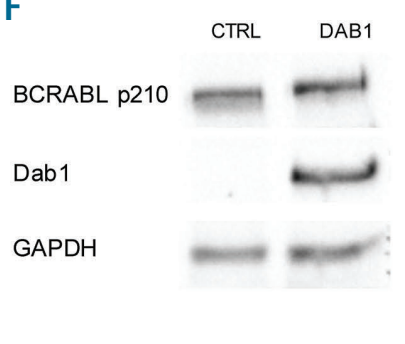

H
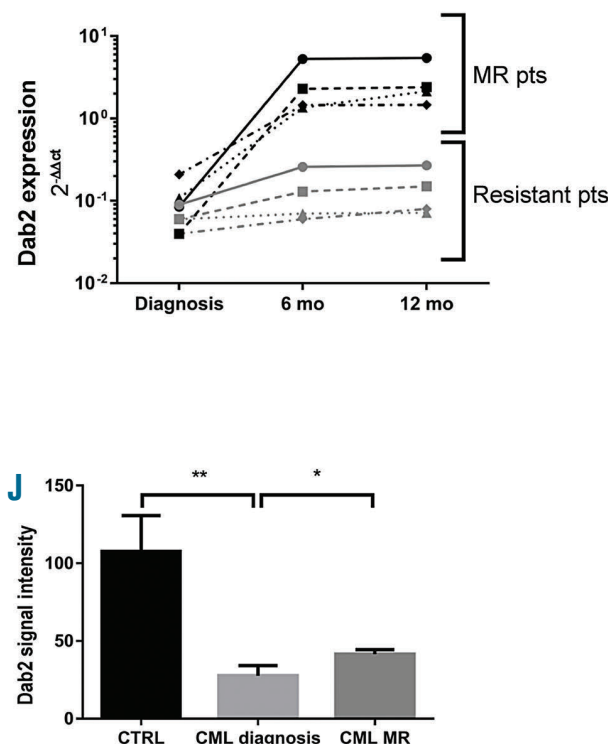

Figure 6. Altered pattern of expression of the human Disabled homologs, Dab1 and Dab2, in patients with chronic myeloid leukemia. (A) Downregulation of Dab1 RNA expression in patients with chronic myeloid leukemia (CML) compared to the expression in healthy donors (CTRL). In particular we found a 1 log reduction of Dab1 expression in both peripheral blood $(\mathrm{PB})(P<0.01)$ and bone marrow (BM) $(P<0.01)$ (median values $2^{-\Delta \Delta c t:} 0.02$ versus 0.3 in $\mathrm{PB}$ and 0.008 versus 0.04 in $\mathrm{BM})$. (B) Expression pattern of Dab1 in CML patients during molecular remission (MR) compared to that in treatment-resistant patients. (C) Immunofluorescence staining of Dab1 protein (red) in PB samples of healthy donors, CML patients at diagnosis and $C M L$ patients during MR. Nuclei are stained in blue. (D) Quantification of Dab1 protein expression in the immunofluorescence assay. (E) A ${ }^{3} \mathrm{H}$-thymidine proliferation assay showing a $20 \%$ reduction of cell proliferation in K562 cells transfected with Dab1 plasmid compared to control. (F) Western blot of protein extracts from K562 cells transfected with an empty vector (lane 1) and transfected with a Dab1 expression vector (lane 2), showing detectable expression of Dab1 only in K562 cells transfected with the Dab1 vector. Independent loads of equal amounts of protein extract were probed with antibodies raised against BCR, Dab1 and GAPDH as a loading control. (G) Down-regulation of Dab2 RNA expression in $\mathrm{CML}$ patients compared to the expression in healthy donors. In particular Dab2 expression was found to be statistically decreased $(P<0.0001$ and $P<0.0001$ in $P B$ and $\mathrm{BM}$, respectively) with median values of 0.12 versus 2.8 and 0.12 versus 0.7 in $\mathrm{PB}$ and $\mathrm{BM}$, respectively. $(H)$ Pattern of expression of Dab2 in CML patients during MR compared to that in treatmentresistant patients. (I) Immunofluorescence staining of Dab2 protein (red) in PB samples of healthy donors, CML patients at diagnosis and $\mathrm{CML}$ patients during MR. Nuclei are stained in blue. (J) Quantification of Dab2 protein expression in an immunofluorescence assay. The statistical comparisons were conducted using a Student $t$ test $\quad(* P<0.05$, $* * P<0.01, * * * * P<0.0001)$. Bars indicate the standard error. 
Moreover, ena-RNAi weakly suppressed the BCR-ABL1 phenotype (Figure 7G), decreasing the phenotype penetrance. As a control, we did not observe any phenotype due to Dab or ena downregulation or Dab overexpression in prohemocytes (Figure 7F,G).

\section{Discussion}

In order to identify candidate genes and pathways involved in the onset and progression of CML we developed and validated a CML genetic model based on trans- genic Drosophila expressing BCR-ABL1. In order to build and characterize a human functional model that could be sensitive to pharmacological inhibition and suitable for studying the effects of BCR-ABL1 mutations identified in patients with CML, we chose to express a completely human $\mathrm{p} 210-\mathrm{BCR}-\mathrm{ABL} 1$ protein, in contrast what has been done previously. The expression of the oncoprotein in all eye cells committed to differentiation as photoreceptors or accessory cells (gmrGal4 driver) induces a strong phenotype characterized by altered differentiation of the ommatidia cells. ${ }^{44}$ The lack of phenotype in flies expressing a BCR-ABL1 kinase-dead mutant sup-
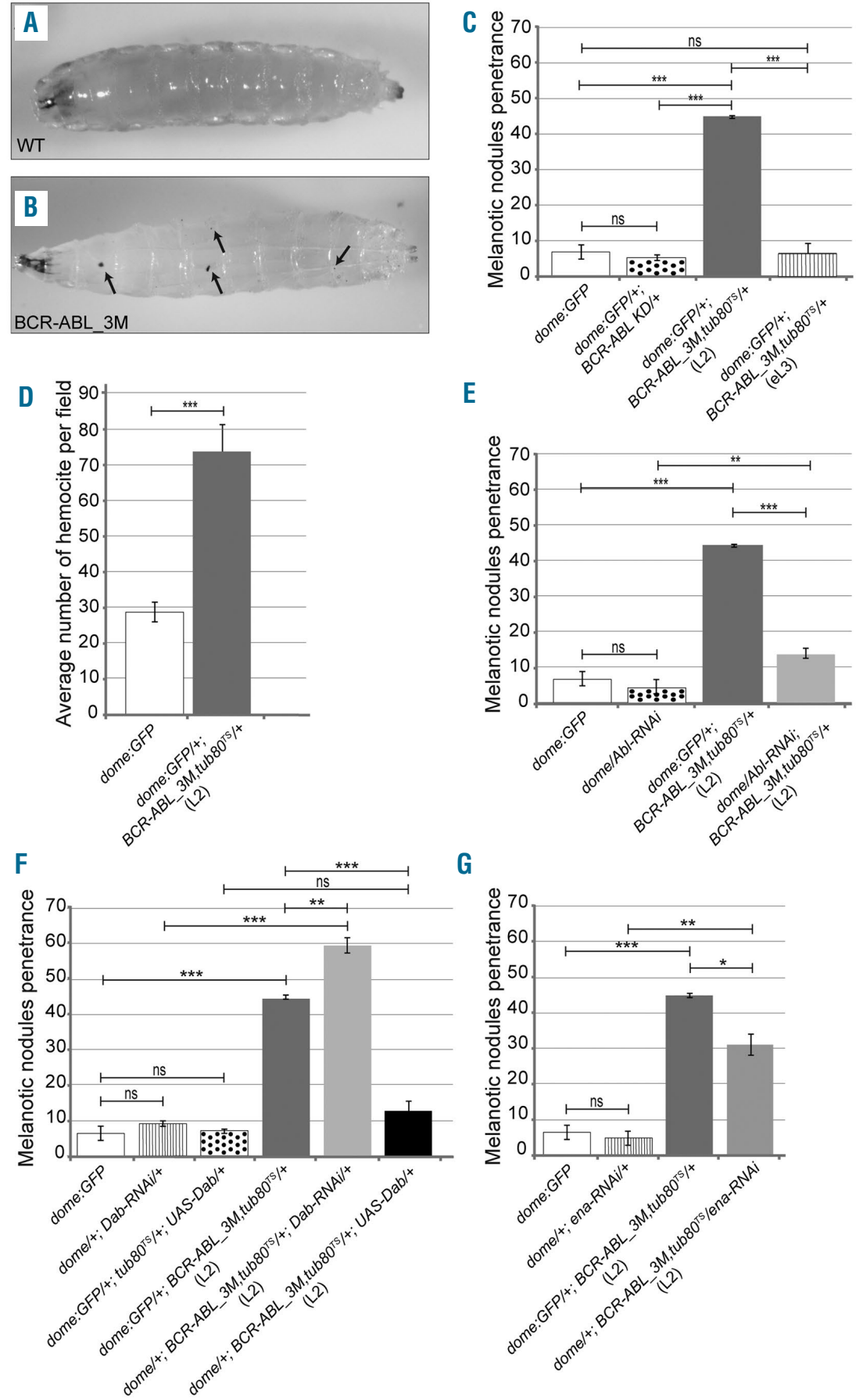

G

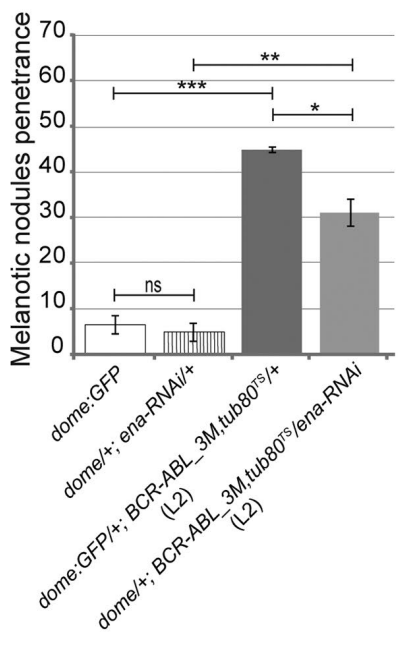

Figure 7. BCR-ABL1 expression in the hematopoietic precursor cells of the lymph gland impairs Drosophila blood cell homeostasis increasing the number of circulating blood cells. (A) A w ${ }^{1118}$ mid-L3 instar larva used as the wild-type control. (B) A mid-L3 larva conditionally expressing BCR-ABL1 in the hematopoietic precursors of the lymph gland medullary zone under the control of the domelessGal4 driver construct (dome:GFP/+;BCR-ABL1_3M,tub80TS/+). BCR$A B L 1$ expression was induced in stage L2 or early-L3 larvae by exposing the animals to $29^{\circ} \mathrm{C}$ during the indicated larval instars to disrupt the ability of the temperature-sensitive Gal80 mutant to inhibit Gal4 transactivation activity. The black arrows in (B) point to melanotic nodules. Anterior is on the left. (C) Penetrance of the melanotic nodule phenotype in mid-L3 contro larvae expressing GFP under the control of the domelessGal4 driver (domeGFP), in larvae constitutively expressing a kinase-dead BCR-ABL1 mutant protein (dome:GFP/+;BCR-ABL1 KD/+) and in larvae in which BCR-ABL1 (dome:GFP/+;BCR-ABL1 3M,tub80TS/+) expression was induced starting from the $L 2(L 2)$ or from the early-L3 (eL3) instars. (D) Evaluation of the average number of hemocytes per field after bleeding of dome:GFP/+;BCRABL1_3M,tub80TS/+ and dome:GFP/+ larvae. BCR-ABL1 expression induces the appearance of melanotic nodules and this correlates with an increase of circulating hemocytes. (E) Penetrance of the melanotic nodule phenotype in mid-L3 control larvae (dome:GFP), in larvae expressing Abl-RNAi (dome/Abl-RNAi), in larvae in which BCR-ABL1 alone (dome:GFP/+;BCRABL1_3M,tub80TS/+) or together with AbI-RNAi (dome/Abl-RNAi;BCR-ABL1_3M,tub80TS/+) is expressed from the $L 2$ instar. $(F)$ Penetrance of the melanotic nodule phenotype in mid-L3 control larvae (dome:GFP), in larvae expressing $D a b$ RNAi (dome/+;Dab-RNAi/+), in larvae conditionally expressing the Dab protein (dome:GFP/+;tub80TS/+:UAS-Dab/+), and in larvae in which BCR-ABL1 alone (dome:GFP/+;BCRABL1_3M,tub80TS/+) or together with either Dab-RNAi (dome/+;BCR-

ABL1 3M,tub80TS/+;Dab-RNAi/+) or UAS-Dab (dome/+;BCR-ABL1_3M,tub80TS/+;UAS-Dab/+) is expressed from the $L 2$ instar. $(G)$ Penetrance of the melanotic nodule phenotype in mid-L3 control larvae (dome:GFP), in larvae expressing ena-RNAi (dome/+;ena-RNAi/+), and in larvae in which BCR-ABL1 alone (dome:GFP/+;BCRABL1_3M,tub80TS/+) or together with ena-RNAi (dome/+;BCR-ABL1 3M,tub80TS/ena-RNAi) is expressed from the $L 2$ instar. The average phenotype penetrance is calculated from three independent experiments, each involving 15-95 larvae. The statistical comparisons were conducted using a Student $t$ test $(* P<0.05, * * P<0.01$ $* * * P<0.001, \mathrm{~ns}=$ not significant). Bars indicate the standard error. 
ports the role of kinase activity in the eye phenotype. Moreover, BCR-ABL1 expression and phosphorylation levels correlate with the severity of the phenotype. Consistently, BCR-ABL1 expression under the control of gmrGaly induces a decrease of photoreceptors expressing Elav in eye imaginal discs and this correlates with the disruption of the adult eye. Interestingly, partial loss of $d A b l$ function also slightly reduces the number of eye cells expressing Elav at L3, and to a much greater extent at later stages of development. This suggests that $d A b l$ is implicated in the maintenance of neuronal commitment $t^{45,46}$ and confirms that loss or gain of function of dAbl/BCR-ABL1 can alter eye cell development.' We have shown that human BCR-ABL1 interacts and interferes with the dAbl signaling pathway. Animals expressing BCR-ABL1 and heterozygous for the recessive $A b l^{\prime}$ allele or coexpressing either Abl-RNAi or a kinase-dead dominant negative $A b l\left(A b I^{K 417 N}\right)$ showed a weaker phenotype, suggesting that BCR-ABL1 and dAbl proteins most likely share binding sites and/or targets of the kinase activity. Consistently, co-expression of human BCRABL1 and dAbl synergizes and the phenotype becomes more severe. Notably, dAbl overexpression per se induces a weak "rough" eye phenotype but the differentiation program is not severely disrupted. We cannot exclude that this is due to a level of dAbl expression below a critic threshold but it could also suggest that excessive dAbl might be still, at least partially, negatively regulated. This possible negative regulation seems to be overcome by BCR-ABL1 since all animals co-expressing $\mathrm{dAbl}$ and BCRABL1 showed a severe class -1 phenotype. Consistently, LOF or downregulation of genes known to interact genetically with $d A b l$ LOF mutations interact in the same way with BCR-ABL1 expression. Namely, pros and fax alleles or deletions enhance the phenotype and this is consistent with their roles in neuronogenesis and neuronal differentiation. Moreover, ena LOF suppresses and Dab LOF enhances the $d A b l$ LOF phenotype ${ }^{19,20}$ and we observed that both ena and Dab LOF and downregulation through RNAi also modify the BCR-ABL1 phenotype in the same way. Ena belongs to the ENA/VASP protein family involved in regulation of the actin cytoskeleton. ${ }^{47,48} \mathrm{dAbl}$ regulates Ena by modulating its localization, most likely through its phosphorylation. It is known that both dAbl and the human/Drosophila BCR-ABL chimera phosphorylate $\mathrm{Ena}^{7}$ in vitro and we established that human BCR-ABL1 expression in the eye also increases Ena phosphorylation. This conservation of phosphorylation targets significantly increases the reliability of our model for identifying relevant BCR-ABL1 functional interactors. In this view the observation that decreased Ena function suppresses phenotypes due to both $d A b l$ mutations ${ }^{24}$ and BCR-ABL1 expression suggests that both phenotypes can be due to Ena mislocalization and consequently actin cytoskeleton alterations can be suppressed if Ena expression decreases. In Drosophila, $A b l$ and Dab are often co-expressed and the phenotype due to Dab mutations mimics the $d A b l$ phenotype. Epistasis experiments have shown that Dab functions upstream of both $\mathrm{dAbl}$ and Ena, controlling their localization and thus the actin cytoskeleton, and Dab LOF does indeed enhance the phenotype due to $d A b l$ mutations. ${ }^{30}$ Interestingly, Dab deletion or downregulation has the same effect on the BCR-ABL1 phenotype. These findings could be explained if $\mathrm{Dab}$ is able to regulate, at least partially, BCR-ABL1 localization. This interaction might mitigate more severe BCR-ABL1-dependent effects when $D a b$ is expressed at a physiological level but not if $D a b$ is downregulated or its gene dosage is halved. Furthermore, our study showed that Dab human homologs are less expressed in both peripheral blood and bone marrow of CML patients at diagnosis compared to their expression in controls and are re-expressed in patients during molecular remission. Moreover, Dab1 expression in transfected K562 cells significantly decreases cell proliferation, confirming that Dab activity might alleviate the pathogenic effects of BCR-ABL1. We then assessed whether our model could help to fish-out homologs of leukemia-relevant genes in an ongoing dosage-sensitive genetic screen of the whole Drosophila genome. To this aim we considered STAT5, a transcription factor phosphorylated and activated by BCR-ABL1. Interestingly, LOF conditions of STAT92E, encoding the fly homolog of various human STAT, led to suppression of the BCR-ABL1 phenotype. In order to discover a tissue that could be a reliable second read-out for identifying BCR-ABL1 interactors relevant for hematopoiesis and leukemia, we moved to the larval hematopoietic organ, the lymph gland. We conditionally expressed human BCR-ABL1 in the lymph gland medullary zone where quiescent prohemocytes reside. Only BCR-ABL1 expression during L2 induces the appearance of melanotic nodules, which correlates with an increase of circulating hemocytes. This phenotype can be suppressed by $d A b l$ downregulation, confirming that $d A b l$ is expressed in the lymph gland medullary zone ${ }^{43}$ where it contributes to BCR-ABL1 pathway activation and to induction of the hematopoietic phenotype. It is worth noting that both $D a b$ and ena interact functionally with BCR-ABL1 during hematopoiesis. In fact, while Dab downregulation enhances the melanotic nodule phenotype and Dab overexpression suppresses it, ena downregulation decreases the penetrance of this phenotype, confirming that ena and Dab are also expressed in the lymph gland medullary zone ${ }^{43}$ and modulate BCR-ABL1 activity. This phenotype is visible if BCR-ABL1 is expressed from the L2, when prohemocytes become quiescent, but not if it is expressed from the early L3, when the quiescent prohemocytes are still present in the medullary zone of the lymph gland. We are tempted to speculate that the dAbl pathway, activated by BCR-ABL1, could be involved in the mechanisms that regulate entry of prohemocytes into the quiescent state rather than maintenance of this state. This seems consistent with the observation that the lymph glands in mid-L3 larvae expressing BCR-ABL1 from L2 are very small compared to those in controls and do not show any clear partition (Giordani and Bernardoni, unpublished data). This suggests that, upon BCR-ABL1 expression, most of the prohemocytes could undertake the differentiation pathway and leave the lymph gland prematurely without becoming quiescent. We did not test all pathways interacting with BCR-ABL1, for example the Tyr-receptor/Ras pathway, which is known to compete with BCR-ABL1 for binding with the Grb2/Drk proteins ${ }^{1}$ and is likely involved in the eye phenotype since the Sevenless Tyr-receptor has an established role in eye differentiation. ${ }^{4,50}$ Nevertheless, we present here a new and efficient CML model based on Drosophila transgenic for human BCR-ABL1. This model could be a powerful tool for identifying new genes and pathways involved in the pathogenesis and progression of CML. 


\section{Acknowledgments}

We thank L. Giardino, V.A. Baldassarro, C. Mangano, and L. Calzà (Fondazione IRET, Ozzano dell'Emilia-Bologna, Italy) for assistance with the confocal microscopy analysis; D. Manzoni and M. Voltattorni for excellent technical help; $M$. Capovilla and the Trans-FlyER, Startup Company, Ferrara, Italy for generating the $B C R-A B L 1$ kinase-dead transgenic lines. Drosophila lines were obtained from the Bloomington Drosophila Stock Center-BDSC (NIH P40OD018537) and primary antibodies from the Developmental Studies Hybridoma Bank (created by the NICHD of the NIH and maintained at The University of Iowa, Department of Biology, Iowa City, IA, USA). We thank the Italian Association for Research on Cancer (AIRC) for funding D. Cilloni (IG10005) and G. Perini (IG11400, IG15182) and for supporting this work.

\section{References}

1. Chereda B, Melo JV. Natural course and biology of CML. Ann Hematol. 2015;94 (Suppl 2):S107-121.

2. Quintas-Cardama A, Cortes J. Molecular biology of bcr-abl1-positive chronic myeloid leukemia. Blood. 2009;113(8):1619-1630

3. Gonzalez C. Drosophila melanogaster: a model and a tool to investigate malignancy and identify new therapeutics. Nat Rev Cancer. 2013;13(3):172-183.

4. Bashaw GJ, Kidd T, Murray D, Pawson T, Goodman CS. Repulsive axon guidance: Abelson and Enabled play opposing roles downstream of the roundabout receptor. Cell. 2000;101(7):703-715.

5. Grevengoed EE, Loureiro JJ, Jesse TL, Peifer M. Abelson kinase regulates epithelial morphogenesis in Drosophila. J Cell Biol. 2001:155(7):1185-1198.

6. Liebl EC, Forsthoefel DJ, Franco LS, et al. Dosage-sensitive, reciprocal genetic interactions between the Abl tyrosine kinase and the putative GEF trio reveal trio's role in axon pathfinding. Neuron. 2000;26(1):107118.

7. Fogerty FJ, Juang JL, Petersen J, Clark MJ, Hoffmann FM, Mosher DF. Dominant effects of the bcr-abl oncogene on Drosophila morphogenesis. Oncogene. 1999;18(1):219-232.

8. Cilloni D, Messa F, Arruga F, et al. The NFkappaB pathway blockade by the IKK inhibitor PS1145 can overcome imatinib resistance. Leukemia. 2006;20(1):61-67.

9. McGuire SE, Mao Z, Davis RL. Spatiotemporal gene expression targeting with the TARGET and gene-switch systems in Drosophila. Sci STKE. 2004;2004 (220):pl6.

10. McGuire SE, Roman G, Davis RL. Gene expression systems in Drosophila: a synthesis of time and space. Trends Genet. 2004:20(8):384-391.

11. Giordani G, Barraco M, Giangrande A, et al. The human Smoothened inhibitor PF04449913 induces exit from quiescence and loss of multipotent Drosophila hematopoietic progenitor cells. Oncotarget. 2016;7(34):55313-55327.

12. Cilloni D, Carturan S, Bracco E, et al. Aberrant activation of ROS1 represents a new molecular defect in chronic myelomonocytic leukemia. Leuk Res. 2013;37(5):520-530.

13. Brand AH, Perrimon N. Targeted gene expression as a means of altering cell fates and generating dominant phenotypes. Development. 1993;118(2):401-415

14. Basler K, Yen D, Tomlinson A, Hafen E. Reprogramming cell fate in the developing Drosophila retina: transformation of $\mathrm{R} 7$ cells by ectopic expression of rough. Genes Dev. 1990;4(5):728-739.
15. Freeman M. Reiterative use of the EGF receptor triggers differentiation of all cell types in the Drosophila eye. Cell. 1996:87(4):651-660

16. Robinow S, White K. The locus elav of Drosophila melanogaster is expressed in neurons at all developmental stages. Dev Biol. 1988;126(2):294-303

17. Gertler FB, Hill KK, Clark MJ, Hoffmann FM. Dosage-sensitive modifiers of Drosophila abl tyrosine kinase function: prospero, a regulator of axonal outgrowth and disabled, a novel tyrosine kinase substrate. Genes Dev. 1993;7(3):441-453.

18. Hill KK, Bedian V, Juang JL, Hoffmann FM Genetic interactions between the Drosophila Abelson (Abl) tyrosine kinase and failed axon connections (fax), a novel protein in axon bundles. Genetics. 1995;141(2):595-606

19. Gertler FB, Bennett RL, Clark MJ, Hoffmann FM. Drosophila abl tyrosine kinase in embryonic CNS axons: a role in axonogenesis is revealed through dosage-sensitive interactions with disabled. Cell. 1989;58(1):103-113.

20. Gertler FB, Doctor JS, Hoffmann FM Genetic suppression of mutations in the Drosophila abl proto-oncogene homolog. Science. 1990;248(4957):857-860.

21. Krause M, Bear JE, Loureiro JJ, Gertler FB. The Ena/VASP enigma. J Cell Sci. 2002;115(Pt 24):4721-4726.

22. Grevengoed EE, Fox DT, Gates J, Peifer M Balancing different types of actin polymerization at distinct sites: roles for Abelson kinase and Enabled. I Cell Biol. 2003;163(6):1267-1279.

23. Comer AR, Ahern-Djamali SM, Juang JL Jackson PD, Hoffmann FM. Phosphorylation of Enabled by the Drosophila Abelson tyrosine kinase regulates the in vivo function and protein-protein interactions of Enabled. Mol Cell Biol. 1998;18(1):152-160

24. Gertler FB, Comer AR, Juang JL, et al enabled, a dosage-sensitive suppressor of mutations in the Drosophila Abl tyrosine kinase, encodes an Abl substrate with SH3 domain-binding properties. Genes Dev. 1995;9(5):521-533.

25. Nieborowska-Skorska M, Wasik MA, Slupianek A, et al. Signal transducer and activator of transcription (STAT) 5 activation by BCR/ABL is dependent on intact Src homology $(\mathrm{SH}) 3$ and $\mathrm{SH} 2$ domains of BCR/ABL and is required for leukemogenesis. J Exp Med. 1999;189(8):1229-1242.

26. de Groot RP, Raaijmakers IA, Lammers JW, Jove R, Koenderman L. STAT5 activation by BCR-Abl contributes to transformation of K562 leukemia cells. Blood. 1999;94(3):11081112

27. Bina S, Wright VM, Fisher $\mathrm{KH}$, Milo $\mathrm{M}$, Zeidler MP. Transcriptional targets of Drosophila JAK/STAT pathway signalling as effectors of haematopoietic tumour formation. EMBO Rep. 2010;11(3):201-207.

28. Zeidler MP, Bach EA, Perrimon N. The roles of the Drosophila JAK/STAT pathway. Oncogene. 2000;19(21):2598-2606.

29. Le N, Simon MA. Disabled is a putative adaptor protein that functions during signaling by the sevenless receptor tyrosine kinase. Mol Cell Biol. 1998;18(8):4844-4854

30. Song JK, Kannan R, Merdes G, Singh J, Mlodzik M, Giniger E. Disabled is a bona fide component of the Abl signaling network. Development. 2010;137(21):3719 3727

31. McAvoy S, Zhu Y, Perez DS, James CD Smith DI. Disabled-1 is a large common fragile site gene, inactivated in multiple cancers. Genes Chromosomes Cancer 2008:47(2):165-174

32. Finkielstein CV, Capelluto DG. Disabled-2: A modular scaffold protein with multifaceted functions in signaling. Bioessays. 2016;38 (Suppl 1):S45-55

33. Zhang Z, Chen Y, Tang J, Xie X. Frequent loss expression of dab2 and promotor hypermethylation in human cancers: a metaanalysis and systematic review. Pak J Med Sci. 2014;30(2):432-437.

34. Holz A, Bossinger B, Strasser T, Janning W Klapper R. The two origins of hemocytes in Drosophila. Development. 2003;130(20): 4955-4962.

35. Evans CJ, Hartenstein V, Banerjee U. Thicker than blood: conserved mechanisms in Drosophila and vertebrate hematopoiesis. Dev Cell. 2003;5(5):673-690.

36. Lanot R, Zachary D, Holder F, Meister M Postembryonic hematopoiesis in Drosophila. Dev Biol. 2001;230(2):243-257.

37. Jung SH, Evans CJ, Uemura C, Banerjee U. The Drosophila lymph gland as a developmental model of hematopoiesis. Development. 2005;132(11):2521-2533.

38. Krzemien J, Dubois L, Makki R, Meister M Vincent A, Crozatier M. Control of blood cell homeostasis in Drosophila larvae by the posterior signalling centre. Nature. 2007;446 (7133):325-328

39. Lebestky T, Jung SH, Banerjee U. A Serrateexpressing signaling center controls Drosophila hematopoiesis. Genes Dev. 2003:17(3):348-353

40. Luo H, Hanratty WP, Dearolf CR. An amino acid substitution in the Drosophila hopTum1 Jak kinase causes leukemia-like hematopoietic defects. EMBO J. 1995;14(7):1412-1420.

41. Minakhina S, Steward R. Melanotic mutants in Drosophila: pathways and phenotypes. Genetics. 2006;174(1):253-263.

42. Bourbon HM, Gonzy-Treboul G, Peronnet F et al. A P-insertion screen identifying novel X-linked essential genes in Drosophila. Mech Dev. 2002;110(1-2):71-83

43. Irving P, Ubeda JM, Doucet D, et al. New insights into Drosophila larval haemocyte 
functions through genome-wide analysis. Cell Microbiol. 2005;7(3):335-350.

44. Ellis MC, O'Neill EM, Rubin GM. Expression of Drosophila glass protein and evidence for negative regulation of its activity in non-neuronal cells by another DNAbinding protein. Development. 1993;119(3): 855-865.

45. Bennett RL, Hoffmann FM. Increased levels of the Drosophila Abelson tyrosine kinase in nerves and muscles: subcellular localization and mutant phenotypes imply a role in cellcell interactions. Development. 1992;116(4):

$$
\text { 953-966. }
$$

46. Xiong W, Morillo SA, Rebay I. The Abelson tyrosine kinase regulates Notch endocytosis and signaling to maintain neuronal cell fate in Drosophila photoreceptors. Development. 2013;140(1):176-184.

47. Barzik M, Kotova TI, Higgs $\mathrm{HN}$, et al Ena/VASP proteins enhance actin polymerization in the presence of barbed end capping proteins. J Biol Chem. 2005;280(31): 28653-28662.

48. Bear JE, Svitkina TM, Krause $M$, et al Antagonism between Ena/VASP proteins and actin filament capping regulates fibroblast motility. Cell. 2002;109(4):509-521.

49. Olivier JP, Raabe T, Henkemeyer M, et al. A Drosophila SH2-SH3 adaptor protein implicated in coupling the sevenless tyrosine kinase to an activator of Ras guanine nucleotide exchange, Sos. Cell. 1993;73 (1):179-191

50. Simon MA, Dodson GS, Rubin GM. An SH3-SH2-SH3 protein is required for p21Ras1 activation and binds to sevenless and Sos proteins in vitro. Cell. 1993;73(1) 169-177. 